\title{
SUPERGRAVITY AND SUPERSTRINGS
}

A Geometric Perspective 
This page is intentionally left blank 


\title{
SUPERGRAVITY AND SUPERSTRINGS
}

\section{A Geometric Perspective}

\section{Vol. 3 : Superstrings}

\author{
Leonardo Castellani \\ Istituto Nazionale di Fisica Nucleare \\ Sezione di Torino
}

\author{
Riccardo D' Auria \\ Dipartimento di Fisica \\ Università di Padova
}

\section{Pietro Fré}

International School for Advanced Studies, Trieste 


\section{Published by}

World Scientific Publishing Co. Pte. Ltd.

P O Box 128, Farrer Road, Singapore 9128

USA office: 687 Hartwell Street, Teaneck, NJ 07666

UK office: 73 Lynton Mead, Totteridge, London N20 8DH

\section{SUPERgRaVITY AND SUPERSTRINGS - A Geometric Perspective}

Copyright @ 1991 by World Scientific Publishing Co. Pte. Ltd.

All rights reserved. This book, or parts thereof, may not be reproduced in any form or by any means, electronic or mechanical, including photocopying, recording or any information storage and retrieval system now known or to be invented, without written permission from the Publisher.

ISBN 9971-50-037-X (set)

ISBN 9971-50-038-8 pbk (set)

Printed in Singapore by Loi Printing Pre. Led. 


\section{CONTENTS}

Preface

\section{Volume 1}

\section{PART ONE. GRAVITY AND DIFFERENTIAL GEOMETRY}

Chapter I.0. Introduction

Chapter I.1. Exterior Calculus

1.1.1. Exterior forms on vector spaces 10

1.1.2. Mappings and operations on forms 23

1.1.3. Differentiable manifolds, vector fields and differential forms 28

1.1.4. Functions, vector fields and differential forms 37

I.1.5. Exterior differentiation and behaviour under mappings 47

1.1.6. The vielbein basis 53

1.1.7. Lie derivative, coordinate transformations and invariance 59

Appendix: The $\delta$ Operator and the Hodge Decomposition 72

Chapter I.2. Riemannian Manifolds $\quad 75$

$\begin{array}{ll}\text { 1.2.1. Introduction } & 75\end{array}$

1.2.2. Geometry of the linear spaces 76

1.2.3. The geometry of general Riemannian manifolds in the vielbein basis 80

1.2.4. Relation with the standard world-iensor formalism 91

Chapter I.3. Group Manifolds and Maurer-Cartan Equations 97

$\begin{array}{ll}\text { 1.3.1. Introduction } & 97\end{array}$

1.3.2. Lie groups as manifolds: left and right invariant voctor fields 98

$\begin{array}{lr}\text { 1.3.3. Maurer-Cartan equations } & 104\end{array}$

$\begin{array}{ll}\text { 1.3.4. Adjoint representation and Killing metric } & 107\end{array}$

$\begin{array}{ll}\text { 1.3.5. Killing metric } & 113\end{array}$

1.3.6. Riemannian geometry of semisimple groups 116

$\begin{array}{ll}\text { 1.3.7. Soft group manifolds } & 119\end{array}$

1.3.8. The example of Poincaré and anti de Sitter soft group manifold 131

Chapter I.4. Poincaré Gravity 141

$\begin{array}{ll}\text { 1.4.1. Poincaré gravity } & 141\end{array}$

1.4.2. Extension to the soft group manifold 152

$\begin{array}{ll}\text { 1.4.3. Building rules for the gravity Lagrangians } & 157\end{array}$

1.4.4. Gravity in de Sitter and anti de Sitter space 166

Chapter 1.5. Coupling of Gravity to Matter Fields 170

1.5.1. Geometrical Lagrangian for scalar fields on a rigid background 170 1.5.2. Extension to the Poincaré group manifold and interpretation
of the Lorentz transformation rules as variational equations 
1.5.3. The interaction of the scalar fields with gravity and the effective cosmological constant

1.5.4. The field equation of a massless scalar field in anti de Sitter space (in general in a curved space)

1.5.5. Geometrical Lagrangian for spin 1 fields

1.5.6. Geometrical Lagrangian for spin $1 / 2$ fields

Chapter 1.6. Differential Geometry of Coset Manifolds

I.6.1. Introduction

1.6.2. Classification of coset manifolds

1.6.3. Coordinates on $\mathrm{G} / \mathrm{H}$ and finite G-transformations 197

$\begin{array}{ll}\text { 1.6.4. Finite transformations on } \mathrm{G} / \mathrm{H} & 204\end{array}$

1.6.5. Infinitesimal transformations and Killing vectors 210

$\begin{array}{ll}\text { 1.6.6. Vielbeins and metric on } \mathrm{G} / \mathrm{H} & 212\end{array}$

$\begin{array}{lr}\text { 1.6.7. Covariant Lie derivative } & 219\end{array}$

$\begin{array}{ll}\text { 1.6.8. Geodesics } & 222 \\ \text { l.6.9. } & 225\end{array}$

$\begin{array}{ll}\text { 1.6.9. Invariant measure } & 225\end{array}$

$\begin{array}{ll}\text { 1.6.10. Connection and curvature } & 226\end{array}$

$\begin{array}{ll}\text { 1.6.11. Rescalings } & 231\end{array}$

1.6.12. A Note on the isometries of $\mathrm{G} / \mathrm{H} \quad 235$

$\begin{array}{ll}\text { 1.6.13. Some examples } & 240\end{array}$

1.6.14. Elements of algebraic topology 254

1.6.15. Homotopy and (co)homology of coset spaces 262

Chapter 1.7. Applications of the Formalism and Miscellaneous Examples

1.7.1. The Brans-Dicke theory 272

1.7.2. Minimal coupling of pseudoscalars through a torsion mechanism 278

1.7.3. The Schwarzschild solution 283

$\begin{array}{ll}\text { Bibliography } & 296\end{array}$

\section{PART TWO. THE ALGEBRAIC BASIS OF SUPERSYMMETRY}

Chapter II.1. Introduction

Chapter II.2. Super Lie algebras, Supermanifolds and Supergroups

II.2.1. The definition of superalgebras and the example of $N$-extended super Poincaré algebra

II.2.2. Classification of the simple superalgebras whose Lie algebra is reductive

$\begin{array}{lll}\text { II.2.3. } & \text { Grassmann algebras } & 333 \\ \text { II.2.4. Supermanifolds } & 338\end{array}$

II.2.5. Supergroups and graded matrices $\quad 345$

II.2.6. Osp $(4 / N)$ as the $N$-extended supersymmetry algebra in anti de Sitter space

Chapter II.3. Super Maurer-Cartan Equations and the Geometry of Superspace

II.3.1. Maurer-Cartan equations of supergroups on supergroup manifolds 360

II.3.2. Maurer-Cartan equations of Osp(4/N) and $\overline{\mathrm{Osp}(4 / N)} 364$ 
11.3.3. Osp(4/N) Maurer-Cartan equations as the structural equations of rigid superspace

II.3.4. Killing vectors on superspace, that is the generators of the supersymmetry algebra of superisometries

Chapter II.4. Poincaré Supermultiplets

II.4.1. How to construct the unitary irreducible representations of the Nextended Poincaré superalgebra

II.4.2. Massive multiplets without central charges 395

II.4.3. Massive multiplets with central charges 411

II.4.4. Massless multiplets $\quad 416$

Chapter II.5. Supermultiplets in Anti de Sitter Space 425

11.5.1. Free field equations and the concept of mass in anti de Sitter space

II.5.2. Unitary irreducible representations of $\mathrm{SO}(2,3)$

II.5.3. Unitary irreducible representations of Osp(4/N) 448

II.5.4. Osp(4/1) supermultiplets 454

II.5.5. Remarks about the $N$-extended case and the example of the Osp(4/2) multiplets

Chapter II.6. Supersymmetric Field Theories: The Example of the Wess-Zumino Multiplet

II.6.1. Supersymmetric field-theories corresponding to an irreducible representation of the supersymmetry algebra

II.6.2. The Wess-Zumino model: the simplest example of a supersymmetric field theory

II.6.3. Superfield interpretation of the Wess-Zumino model and iheonomy

II.6.4. The integrability of the heonomic conditions and the Bianchi identities

II.6.5. The theonomic action principle

Chapter II.7. $\Gamma$-matrix Algebra and Spinors in $4 \leq D \leq 11$

II.7.1. The construction of $\Gamma$-matrices

II.7.2. The charge conjugation matrix

II.7.3. Majorana, Weyl and Majorana-Weyl spinors 526

II.7.4. Useful formulae in $\Gamma$-matrix algebra

Chapter II.8. Fierz Identities and Group Theory 535

II.8.1. Introduction $\quad 535$

11.8.2. The structure of forms on $N$-extended $D=4$ superspace 537

11.8.3. Fierz decompositions in the $N=1, D=4$ superspace 545

11.8.4. The $N=2, D=4$ case 547

II.8.5. The $N=3, D=4$ case 552

II.8.6. The $N=2, D=5$ case 555

II.8.7. Systematics of Fierz identities in eleven dimensions 563

II.8.8. Irreducible representations of $\mathrm{SO}(1,9)$ and the irreducible basis of the $D=10$ superspace 
viii

Chapter II.9. Super Yang-Mills Theories 582

11.9.1. Introduction 582

11.9.2. Super Yang-Mills theories in $D=4$

11.9.3. The action principle for $N=1, D=10$ super Yang-Mills theory 594

Historical Remarks and References $\quad 597$ 


\section{Volume 2}

\section{PART THREE. SUPERGRAVITY IN THE RHEONOMY FRAMEWORK}

Chapter III.1. Introduction

Chapter III.2. Supergravity in the Standard Component Approach

III.2.1. Local supersymmetry and gravity

III.2.2. Space-time Lagrangian of $D=4, N=1$ supergravity

1II.2.3. The equations of motion of $D=4, N=1$ supergravity

III.2.4. Supersymmetry transformations and action invariance

III.2.5. On-shell supersymmetry invariance

III.2.6. The linearized theory of supergravity

Appendix III.2.A. Commutator of Two Supersymmetries on the Gravitino Fiold

Chapter III.3. Supergravity in Superspace and the Rheonomy

$$
\text { Principle }
$$

III.3.1. From space-time to superspace

III.3.2. Goometry of superspace

III.3.3. The theonomy principle

III.3.4. An extended action principle

III.3.5. $D=4, N=1$ supergravity and theonomy

665

III.3.6. Rheonomic constraints and Bianchi identities 672

III.3.7. On-shell supersymmetry

III.3.8. Action invariance and off-shell supersymmetry 680

III.3.9. Building rules for supergravity Lagrangians

III.3.10. Retrieving $N=1, D=4$ supergravity from the building rules

III.3.11. Extension to anti de Sitter supergravity

III.3.12. Building rules for supergravity theories using ineonomy and Bianchi identities

Chapter III.4. $D=4, N=2$ Simple Supergravity

III.4.1. Introduction

III.4.2. Rheonomic solution of the $N=2, D=4$ Bianchi identities

III.4.3. The Lagrangian of $N=2, D=4$ supergravity

Chapter III.5. The $D=5, N=2$ Supergravity Theory

III.5.1. Introduction

III.5.2. Identification of the supergroup and construction of its curvatures 758

III.5.3. Construction of the Lagrangian

III.5.4. Superspace equations of motion and on-shell supersymmetry 777

III.5.5. The second order formulation and the contracted version of the theory

Chapter III.6. The Theory of Free Differential Algebras and

$$
\text { Some Applications }
$$

III.6.1. Introduction

III.6.2. The concept of free differential algebra

III.6.3. The structure of free differential algebras and some theorems 
III.6.4. Gauging of the free differential algebras and the building rules revisited

III.6.5. The Sohnius-West model (new minimal $N=1$ supergravity): the on-shell formulation

III.6.6. The Sohnius-West model: off-shell extensions 817

III.6.7. The building rules in their final form 826

Chapter III.7. Supergravity in 6 Dimensions 832

III.7.1. Introduction $\quad 832$

III.7.2. $D=6$ Weyl spinors and selfdual tensors 834

III.7.3. The free differential algebra of $D=6$ supergravity 841

III.7.4. Construction of the model $\quad 844$

III.7.5. Non-invariance of the space-time action and how to cure it 855

Chapter III.8. $D=11$ Supergravity 861

III.8.1. Introduction 861

III.8.2. Free differential algebra of $D=11$ supergravity 863

III.8.3. Extended F.D.A. and the introduction of a 6-form 866

III.8.4. The gauging of F.D.A. revisited 868

III.8.5. Constructing the theory from Bianchi identities 873

III.8.6. The action of $D=11$ supergravity 881

III.8.7. The completion of the action and the equations of motion 897

Historical Remarks and References

\section{PART FOUR. THE ROLE OF THE SCALAR FIELDS: $\sigma$-MODEL AND SUPERHIGGS PHENOMENON IN SIMPLE AND EXTENDED SUPERGRAVITY}

Chapter IV.1. Introduction

Chapter IV.2. Kähler manifolds

IV.2.1. $\sigma$-models of supergravity and complex manifolds

IV.2.2. Almost complex and complex structures on a $2 n$-dimensional manifold

IV.2.3. Hermitean and Kähler metrics

IV.2.4. The differential geometry of Kähler manifolds

Chapter IV.3. Coupling of $N=1$ Supergravity to $n$ Scalar Multiplets

IV.3.1. Kähler geometry for the $N=1$ coupling

IV.3.2. Solution of the Bianchi identities and auxiliary fields

IV.3.3. Construction of the action: generalities

IV.3.4. Construction of $\mathscr{L}^{\text {(inv) }}$

IV.3.5. Construction of $\Delta \mathscr{L}$

Chapter IV.4. The Vector Multiplets and the Gauging of the Kähler Manifold Isometries

IV.4.1. Killing vectors and isometries of the scalar manifold 
Chapter IV.5. The Super Higgs Phenomenon

$\begin{array}{ll}\text { IV.5.1. Introduction } & 997\end{array}$

IV.5.2. The mass relation in the minimal coupling case 1003

IV.5.3. Examples and flat potentials

1013

Chapter IV.6. Duality Transformations and the Coset Structure of Extended Supergravities

1018

IV.6.1. How to extend the symmetries of the non linear $\sigma$-model to the vector fields

1018

IV.6.2. The coset structure of extended supergravities in $D=4 \quad 1030$

Chapter IV.7. The Example of the $N=3$ Theory

1033

IV.7.1. Introductory remarks

1033

IV.7.2. The $N=3$ vector multiplet and the $G / H$ structure of the supergravity coupling

1034

IV.7.3. $S U(3, n) / S U(3) \otimes S U(n) \otimes U(1)$ formalism and the solution of Bianchi identities

IV.7.4. The Lagrangian

Appendix A: The Scalar Potential

Appendix B: The $A_{N \Sigma}$ Matrix and the Embedding of $S U(3, N)$ into the Symplectic Group

Chapter IV.8. The Supersymmetry Breaking in the $N=3$ Theory and a Short Account of the $N=4$ Theory

IV.8.1. Introduction to partial supersymmetry breaking

IV.8.2. Features of the $\mathbf{N}=\mathbf{3}$ theory and of its potential

IV.8.3. A short discussion of simple $\mathbf{N}=\mathbf{4}$ supergravity

IV.8.4. A short discussion of matter coupled $N=4$ supergravity

Chapter IV.9. The Directory of Supergravity Theories and the $N=8$ Model

IV.9.1. Introduction

IV.9.2. Classification of $D=4$ supergravities and guide to the related literature

IV.9.3. The $N=8$ Theory

IV.9.4. Results for matter coupling in $D=4$ supergravities

References

\section{PART FVE. KALUZA-KLEIN SUPERGRAVITY}

Chapter V.1. Introduction

Chapter V.2. Spontaneous Compactification of $D=5$ Pure Gravity

V.2.1. Spontaneous compactification of $D=5$ pure gravity

V.2.2. Symmetries in $D=4$

V.2.3. A preliminary example: the spectrum of $M_{4} \times S^{1}$ Maxwell theory

V.2.4 The Spectrum of $M_{4} \times S^{\prime}$ gravity 
V.3.2. Harmonic expansions in Kaluza-Klein theories 1182

V.3.3. Yang-Mills fields from $M_{4} \times M_{k}$ compactifications 1186

Chapter V.4. Compactifying Solutions of $D=11$ Supergravity 1189

V.4.1. The $D=4$ vacuum: maximal symmetry 1189

V.4.2. AdS $^{4} \times M^{7}$ solutions (Freund-Rubin) 1192

$\begin{array}{ll}\text { V.4.3. Properties of the internal space } M^{7} \text { : Killing spinors and Weyl } & 1194 \\ \text { holonomy } & \end{array}$

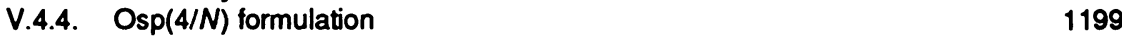

$\begin{array}{ll}\text { V.4.5. Differential operators on } M^{7} & 1204\end{array}$

$\begin{array}{ll}\text { Appendix V.4.1. SO(7) I-matrices } & 1217\end{array}$

Chapter V.5. The $D=4$ Mass Spectrum in $\mathrm{AdS}^{4} \times M^{7}$ Backgrounds $\quad 1221$

V.5.1. The linearized field equations of $D=11$ supergravity 1221

V.5.2. Fermion masses 1224

V.5.3. Boson masses 1230

V.5.4. Supersymmetric mass relations 1232

$\begin{array}{ll}\text { V.5.5. Vacuum stability } & 1241\end{array}$

Chapter V.6. Classification of Compact Homogeneous $D=7$ Einstein Spaces

V.6.1. Homogeneous 7-manifolds 1249

V.6.2. The spaces $S U(3) \times S U(2) \times U(1) / S U(2) \times U(1) \times U(1)$

V.6.3. The other $D=7$ Einstein spaces $G / H \quad 1254$

Chapter V.7. The Spectra of Specific Solutions: The Seven-Sphere 1259

V.7.1. How to compute $\mathscr{D}$ on the $G / H$ harmonics 1259

V.7.2. The spectrum of the round $S^{7}$ : harmonic analysis 1262

V.7.3. The spectrum of the round seven-sphere: Osp(4/8) analysis 1277

Chapter V.8. The $M^{p q}$ spaces 1302

V.8.1. The Mpa spaces 1302

V.8.2. Harmonics on the Mpa spaces 1306

V.8.3. The spectrum of the $S U(3) \times S U(2) \times U(1)$ irreps in the

V.8.4. Conjugation in the longitudinal spectrum 1322

V.8.5. Calculation of the longitudinal mass eigenvalues 1329

Chapter V.9. Other Classical Solutions of $D=11$ Supergravity 1343

V.9.1. Introduction 1343

V.9.2. Nonvanishing internal photon (Englert-type solutions) 1343

$\begin{array}{ll}\text { V.9.3. Symmetries of Englert-type solutions } & 1347\end{array}$

V.9.4. Stretched and warped solutions 1347

Chapter V.10. The Embedding of $D=4$ S.G. into $D=11$ S.G. 1355

Chapter V.11. The Chirality Problem 1360

$\begin{array}{ll}\text { Bibliographical Note } & 1367\end{array}$ 


\section{Volume 3}

\section{PART SIX. HETEROTIC SUPERSTRINGS AND SUPERGRAVITY}

Chapter VI.1. Introduction

Chapter VI.2. Elements of Two-dimensional Differential Geometry and of Riemann Surface Theory

VI.2.1. Introduction

VI.2.2. Definition of a Riemann surface; metrics, complex structures and moduli space

VI.2.3. The simply connected Riemann surfaces and the uniformization theorem

VI.2.4. Deformation of the metric, quadratic differentials and the complex structure of Teichmüller space

VI.2.5. Homology bases, abelian differentials and the period matrix

VI.2.6. Dehn twists, the mapping class group and its homomorphism onto $\mathrm{Sp}(2 \mathrm{~g}, \mathrm{Z})$

VI.2.7. The group of divisors and the Riemann-Roch theorem

VI.2.8. The Jacobian variety: Riemann theta functions and spin structures

Chapter VI.3. The Classical Action of the Heterotic Superstrings and Their Canonical Quantization

VI.3.1. Introduction

VI.3.2. $N=1, D=2$ conformal supergravity and the heterotic superspace geometry

VI.3.3. Classical superconformal theories and the WZW-action

VI.3.4. Heterotic $\sigma$-model on a general target space and the choice of $M_{\text {turen }}$ leading to a classical superconformal theory

VI.3.5. Canonical quantization of the heterotic WZW-model and the superconformal algebra

Appendix: Rules for the Wick Rotation of Spinors 1556

Chapter VI.4. The BRST Charge and the Ghost Fields 1558

VI.4.1. Introduction 1558

VI.4.2. BRST quantization. Abstract properties of Q 1559

VI.4.3. Construction of $Q \quad 1561$

VI.4.4. The BRST invariant hamiltonian and the Fradkin-Vilkovski theorem

VI.4.5. BRST quantization of string theories

Chapter VI.5. Quantum Determination of the Target Manifold and Kac-Moody Algebras

VI.5.1. Introduction

VI.5.2. The BRST charge: cancellation of the conformal anomaly, boundary conditions and intercepts

VI.5.3 Twisted Kac-Moody algebras and massless target fermions

Chapter VI.6. The Polyakov Path Integral and the Partition Function of String Models 
VI.6.2. The cosmological constant, the partition function and the Polyakov path integral

VI.6.3. Operatorial evaluation of the bosonic string partition function 1646

VI.6.4. The Polyakov integration measure for the bosonic string 1654

VI.6.5. Functional evaluation of the bosonic string partition function in the case of the torus

VI.6.6. Functional determinants of the Laplacian and of the Dirac operator on the torus

V1.6.7. The gravitino ghost

Appendix. A Detailed Treatment of Conformal Killing Vectors

Chapter IV.7. Modular Invariance, Fermionization and the Particle Spectrum of Heterotic Superstrings

VI.7.1. Introduction

VI.7.2. Modular invariance and GNO fermionization

VI.7.3. Modular invariance and spin structures

VI.7.4. An example in $D=10$ : the $S O(32)$ superstring

VI.7.5. A second example in $D=10$ : the $E_{8} \otimes E_{8}^{\prime}$ and $S O(16) \otimes S O(16)$ heterotic strings

VI.7.6. Examples in $D=4$

Chapter VI.8. Quantum Conformal Field Theories, Vertex Operators and String Tree Amplitudes

VI.8.1. Introduction

VI.8.2. Quantum conformal field theories and emission vertices

VI.8.3. Bosonization, vertex operators and spin fields in the matter sector

VI.8.4. b-c systems, superghost bosonization and the background charge

VI.8.5. The covariant lattice for $D=10$ superstrings

VI.8.6. Conjugacy dasses and GSO projectors: the SO(32) example in $D=10$

VI.8.7. Massless emission vertices and the effective theory of $D=10$ superstrings

Chapter VI.9. Effective Supergravity Theories and the Coupling of the Lorentz Chern-Simons Term

VI.9.1. Introduction

VI.9.2. The algebraic basis of $N=1, D=10$ matter-coupled supergravity

VI.9.3. The general solution of the $D=10$ super Poincaré Bianchi identities

VI.9.4. The $H$-Bianchi identity in the $(0,4)$ - and $(1,3)$-sectors: determination of

VI.9.5. The $(2,2)$ - and $(3,1)$-sectors of the $\mathscr{H}$-Bianchi identity and the equations of motion

VI.9.6. The Lagrangian of $N=1, D=10$ matter-coupled supergravity at $\gamma=0$

VI.9.8. Bianchi identities and off-shell formulations of $N=1, D=4$ supergravity revisited

VI.9.9. Chiral multiplets, the linear multiplet and the geometrical interpretation of R-symmetry

VI.9.10. $D=4$ Chem-Simons cohomology and the linear multiplet 
Chapter VI.10. $(2,2)$ Superconformal Field Theories and the

Classification of $N=1, D=4$ Heterotic Superstring Vacua

VI.10.1. Introduction 2028

VI.10.2. Type II superstrings on SU(2) ${ }^{3}$ groupfolds

2032

VI.10.3. Construction of modular invariants and GSO projectors for the type II superstring

VI.10.4. SU(2) ${ }^{3}$ groupfolds and superconformal field theories

2039

VI.10.5. The h-map

2050

VI.10.6. Emission vertices of the massless multiplets in an $N=1$ heterotic

2056

VI.10.7. Emission vertices of the massless multiplets in $N=2$ heterotic models based on $a(4,4)_{10} \oplus(2,2)_{33}$ internal theory

VI.10.8. Embedding of a $(2,2)_{9,}$ into the direct sum $(4,4)_{0,6} \oplus(2,2)_{3,3}$

VI.10.9. Classification of the SU(2) ${ }^{3}$ groupfold realizations of the internal conformal field theory

2078

VI.10.10. Details of the SU(2) ${ }^{3}$ groupfold construction with emphasis on bosonization

2083

Appendix VI.10.A. A bosonizable $(2,2)$ vacuum of type A:

A1 $(1,2,3,4) \epsilon_{13}$

2091

Appenidx VI.10.B. A Bosonizable $(2,2)$ Vacuum of Type B:

$B 5(1,2,3,4) \epsilon_{20}$

2094

Appendix VI.10.C. An LRP $(2,2)$ Vacuum of Type B: B26(1,2,3) 2097

Bibliographical Note

Historical Remarks and References

Index 